\title{
Embedding S = 1/2 Kagome-like Lattice in Reduced Graphene Oxide
}

\section{Kriti Gupta}

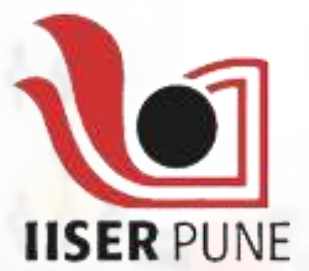




\section{Kagome}

Japanese Basket

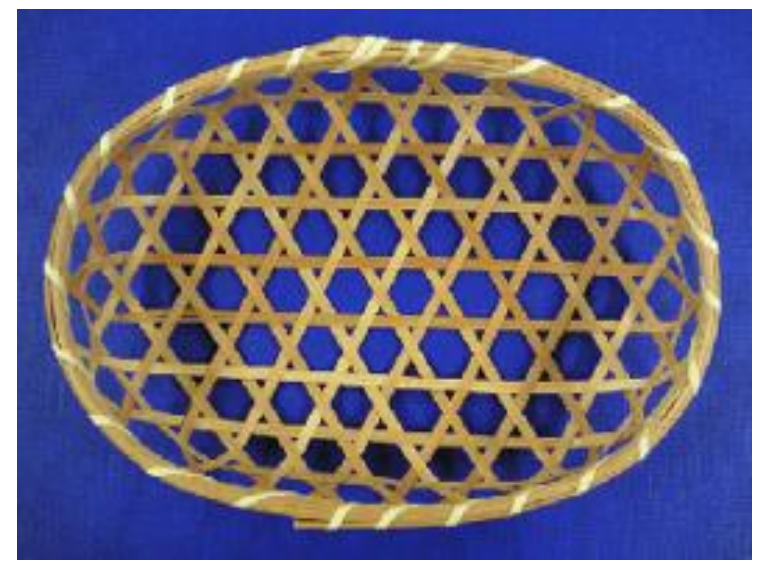

Corner sharing triangles
Kagome Lattice

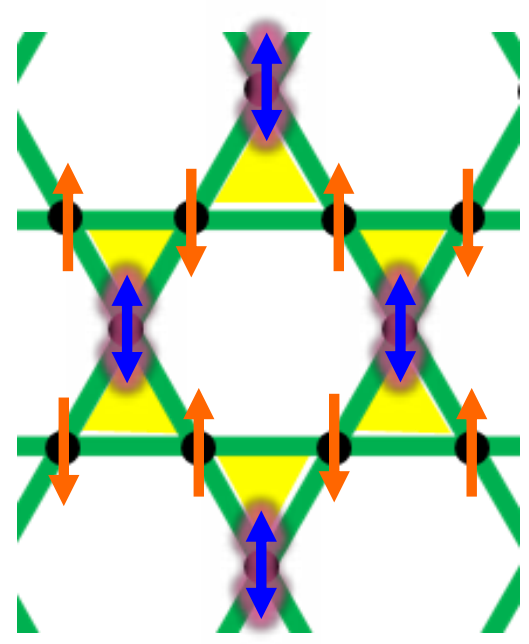

Possible Spin Liquid
Clinoatacamite

$\mathrm{Cu}_{2}(\mathrm{OH})_{3} \mathrm{Cl} ; \mathrm{S}=1 / 2$

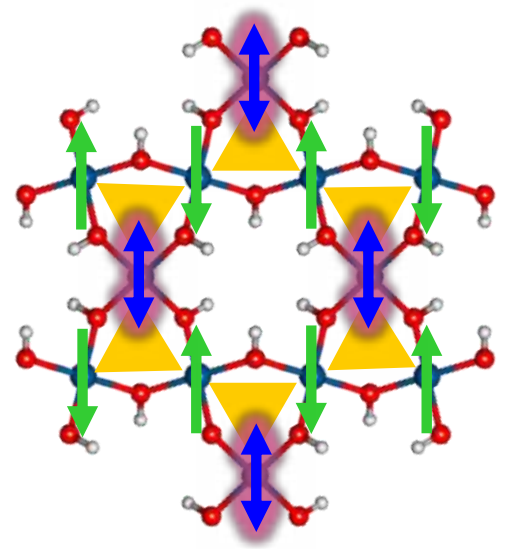

- $\mathrm{Cu}$

- 0

O H
Naturally occurring mineral Possible Quantum Spin Liquid
Inter kagome site $(S=1 / 2)$ eg. $\mathrm{Cu}(\mathrm{II})$ Kagome-like lattice
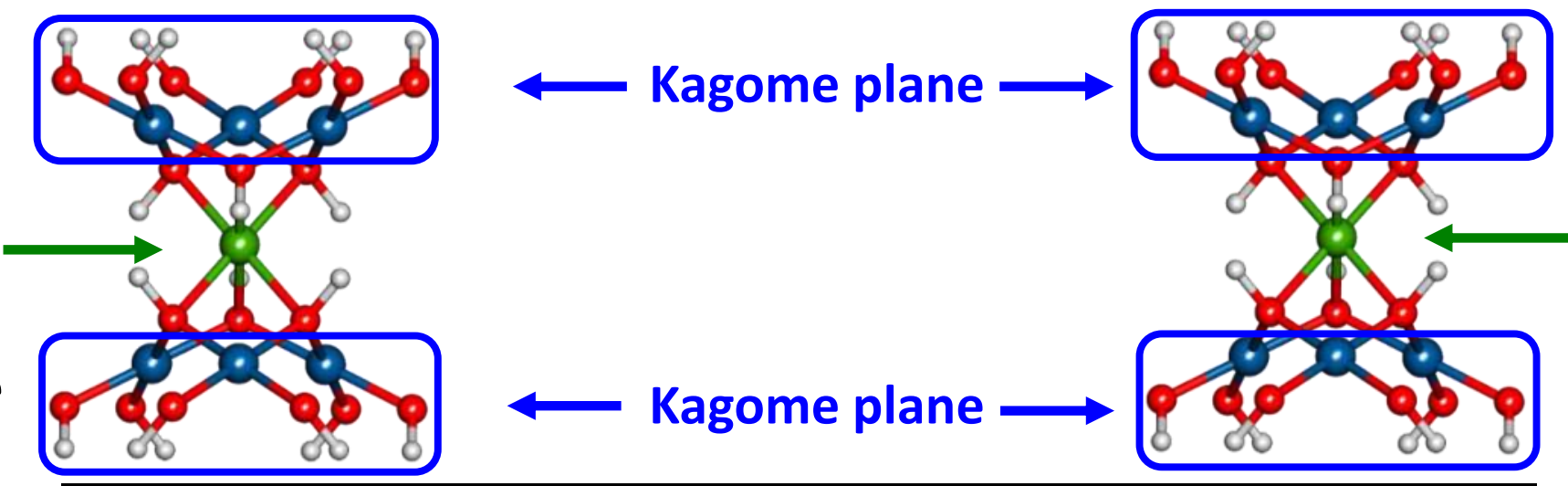

Inter kagome site $(S=0)$ eg. Zn(II) Kagome lattice

What if this frustration is perturbed with a semiconductor..? 


\section{Reduced Graphene Oxide}

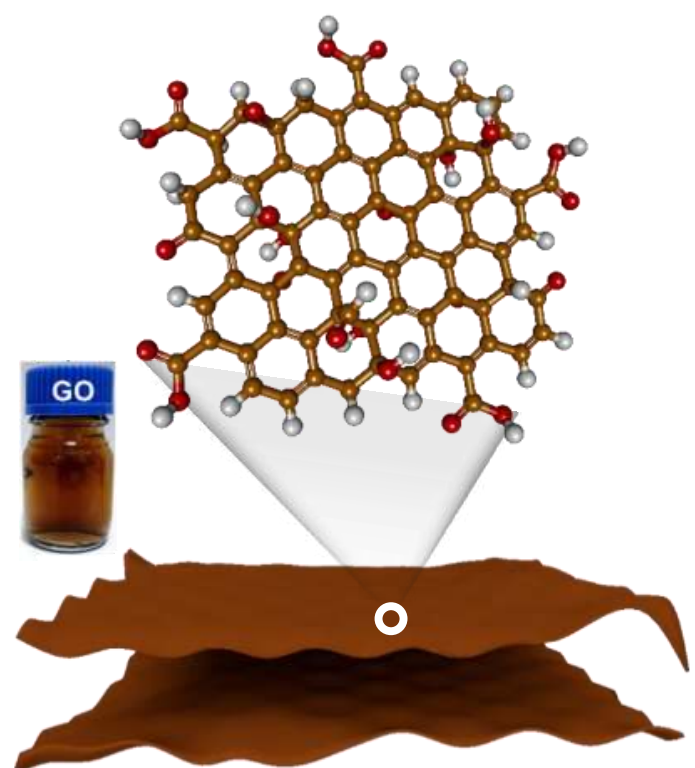

Graphene oxide (GO)

- Carbon

- Oxygen

C Hydrogen
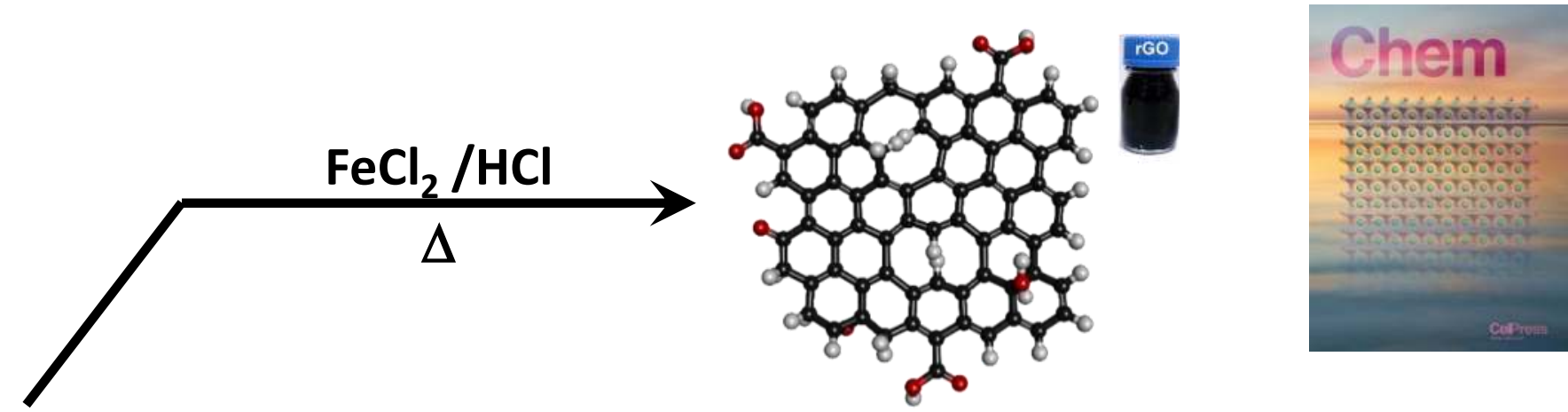

Reduced graphene oxide (rGO)

Chem 2017, 3, 846-860

Indian Patent Application Number:

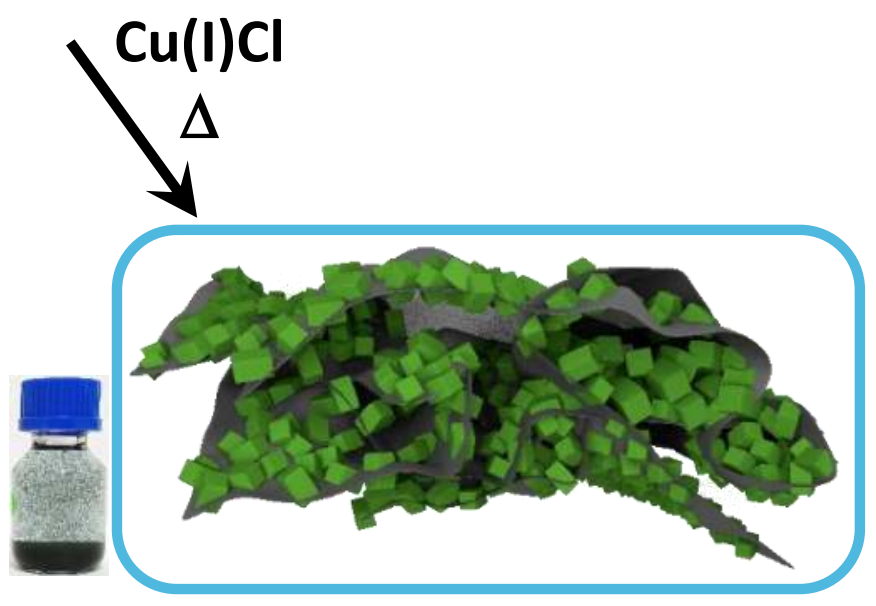

$$
201621023063
$$

- Carbon

- Oxygen

O Hydrogen

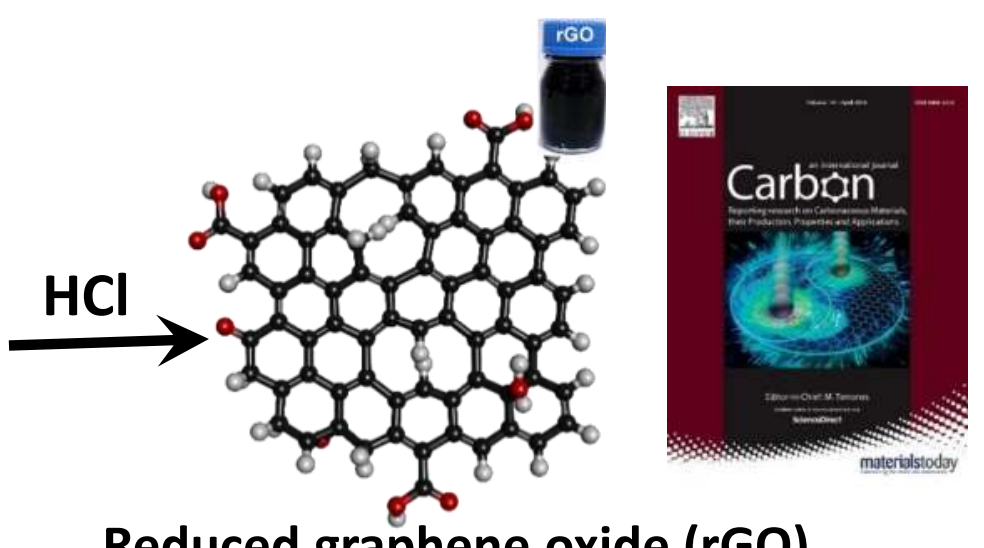

Reduced graphene oxide (rGO)

Carbon 2019, 148, 354-360 


\section{6

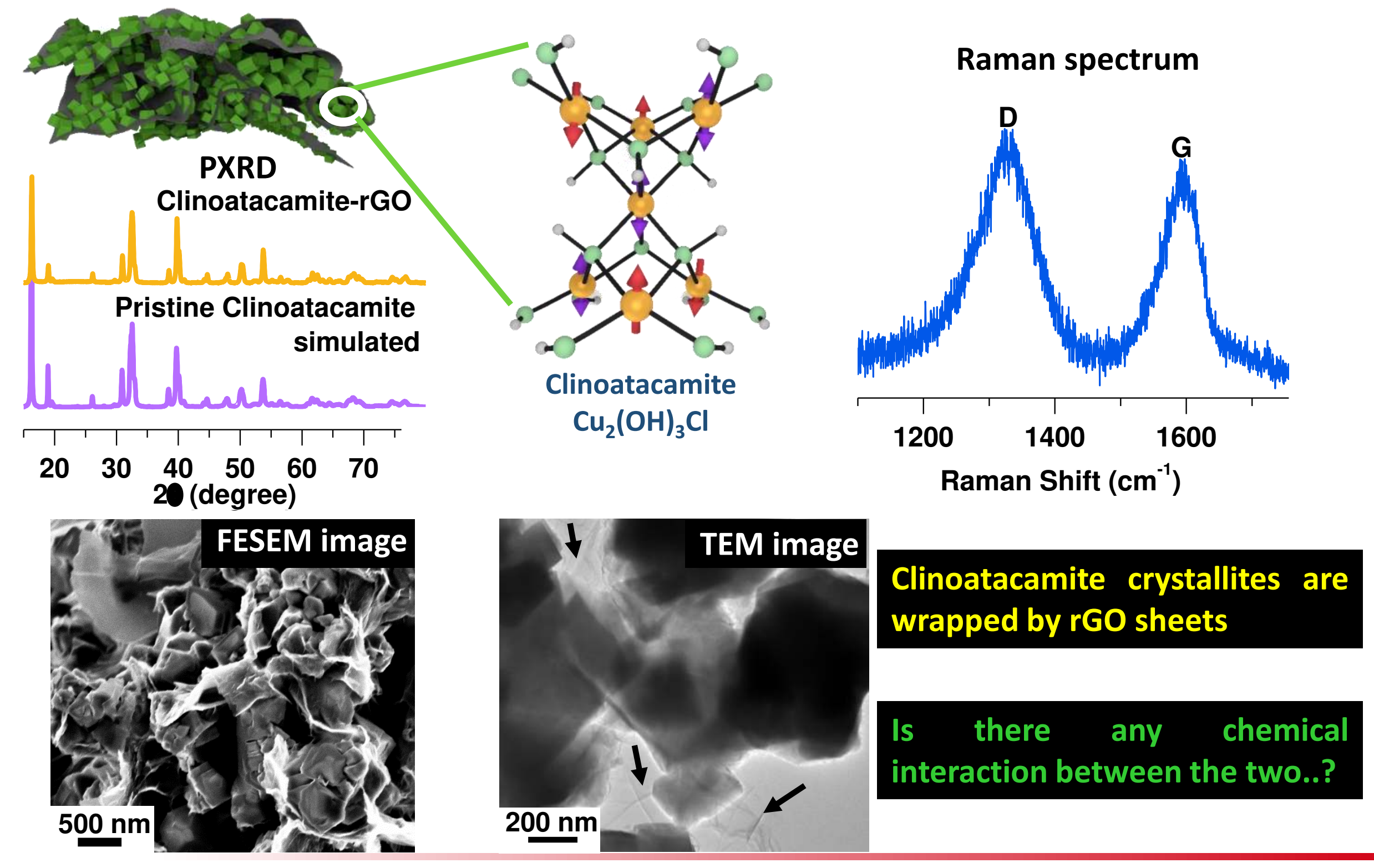




\section{Clinoatacamite-rGO Interaction}

\section{1s XPS spectra}
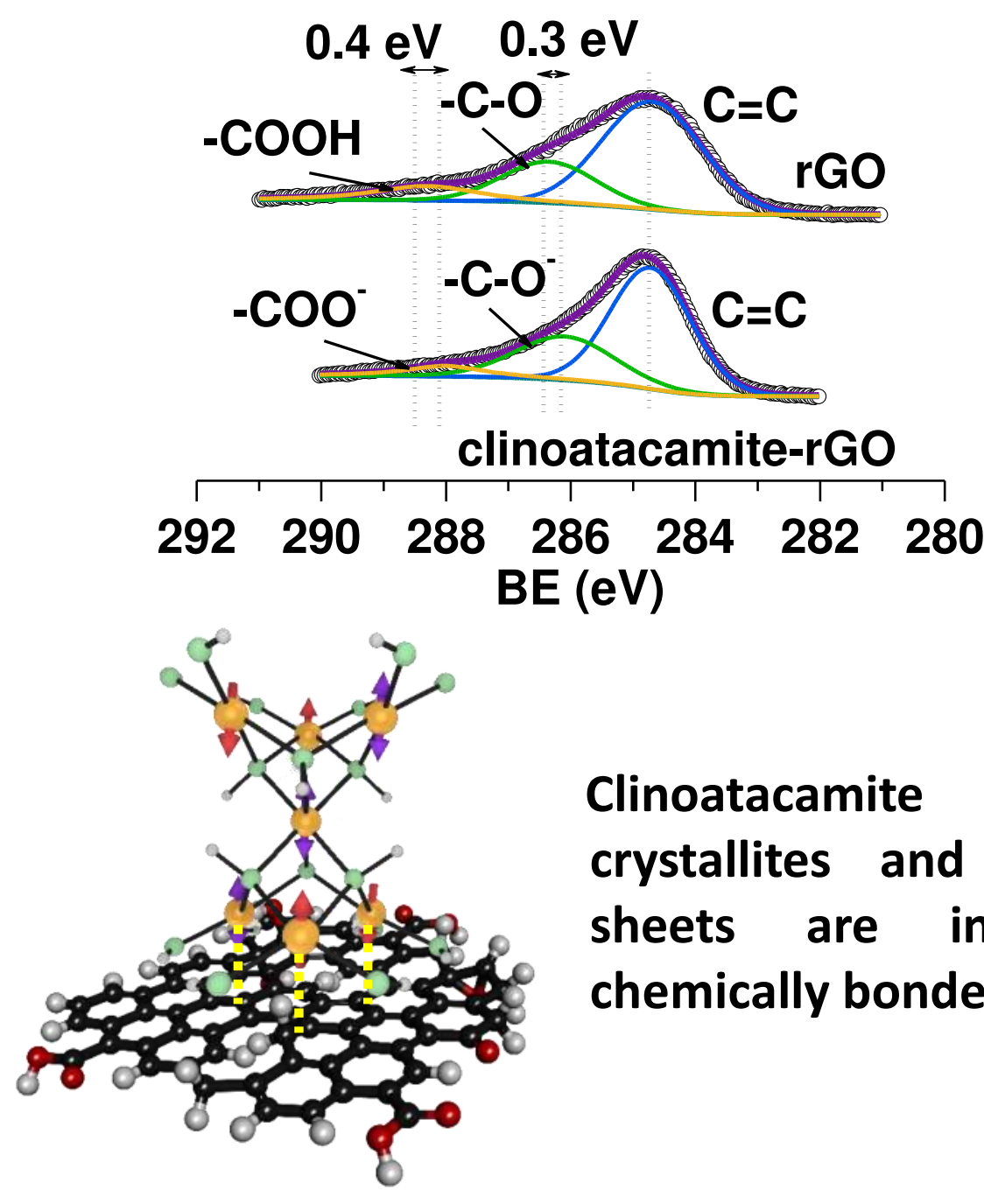

Clinoatacamite crystallites and rGO sheets are indeed chemically bonded
Cu 2p XPS spectra
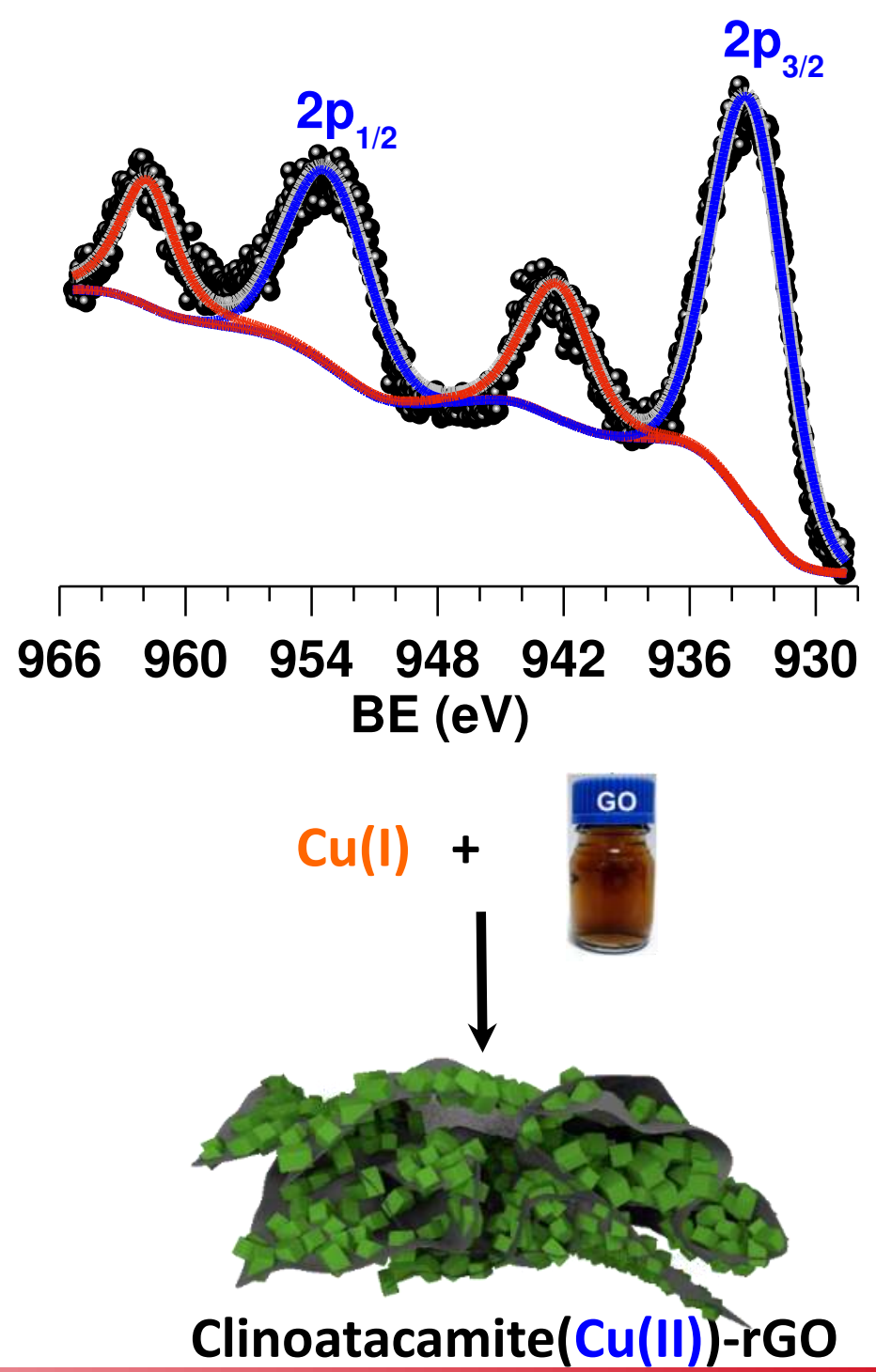


\section{Pristine Clinoatacamite}

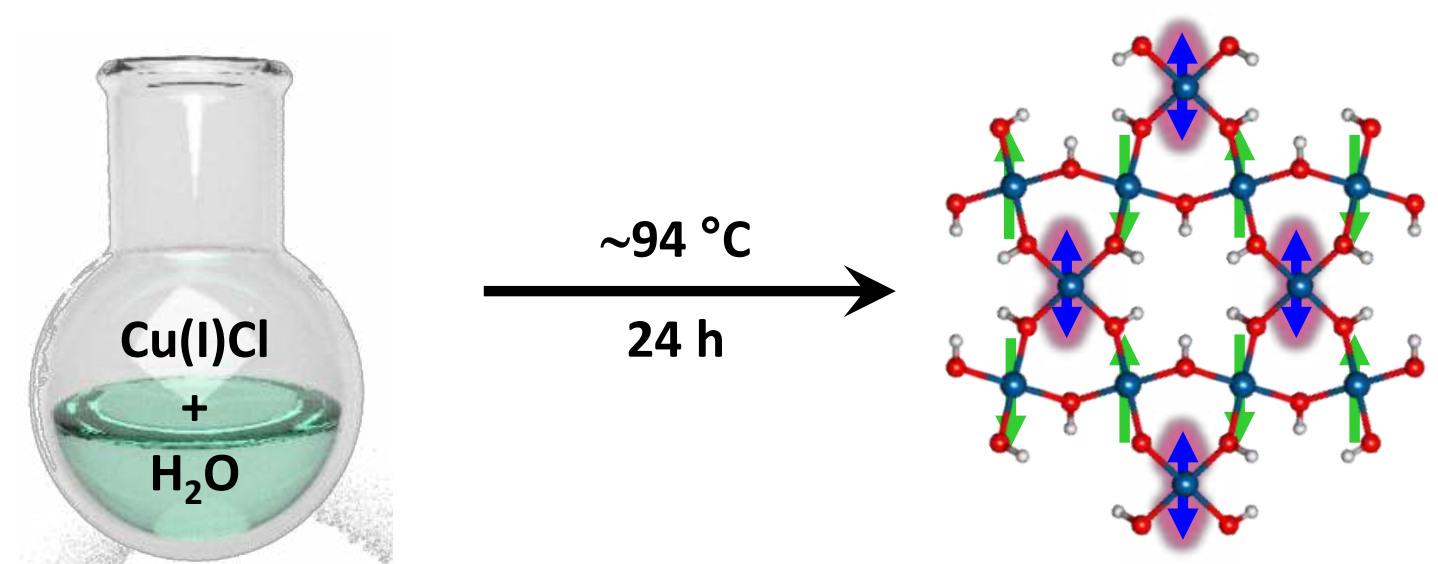

Pristine clinoatacamite
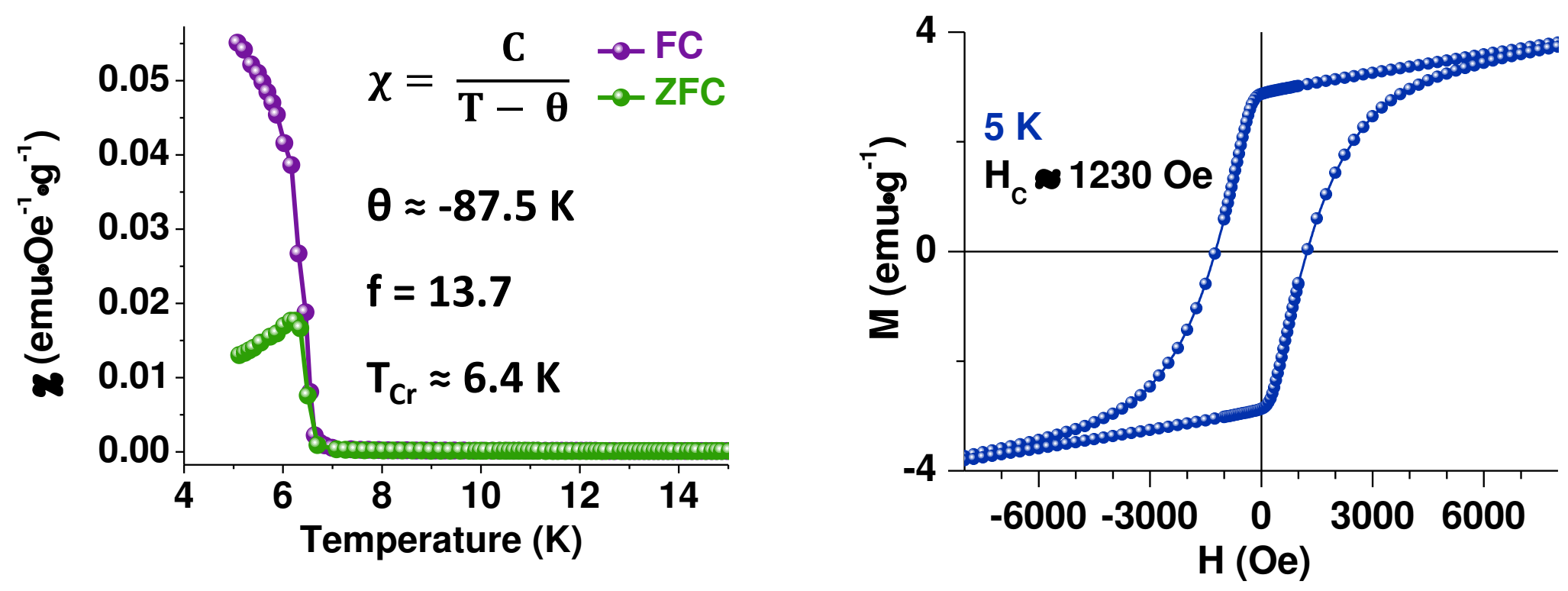

FC - field cooled; ZFC - zero field cooled modes 


\section{Magnetic Response - Nanocomposite}
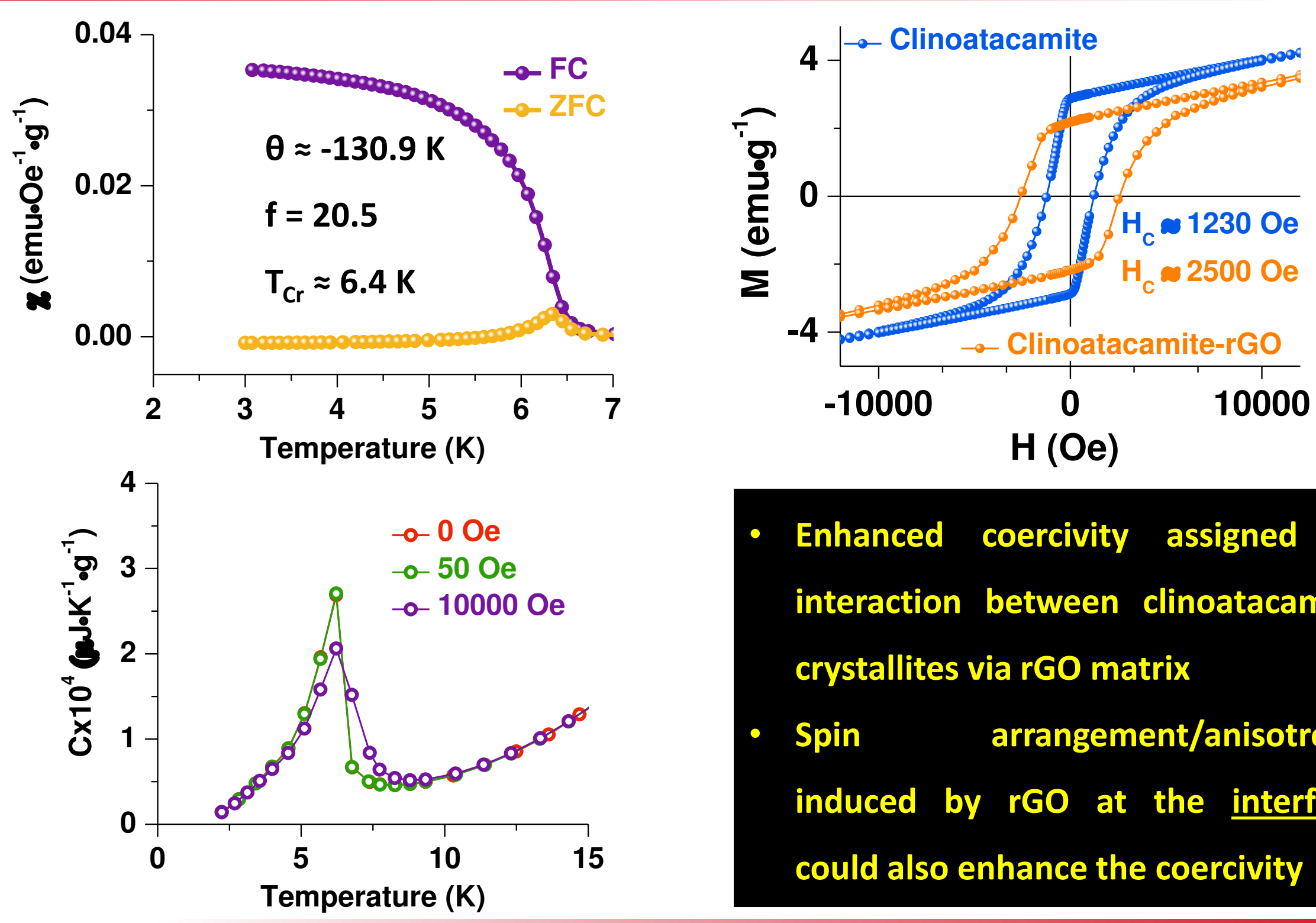

- Enhanced coercivity assigned to interaction between clinoatacamite crystallites via rGO matrix

- Spin arrangement/anisotropy induced by rGO at the interface could also enhance the coercivity 


\section{Electrical Response}
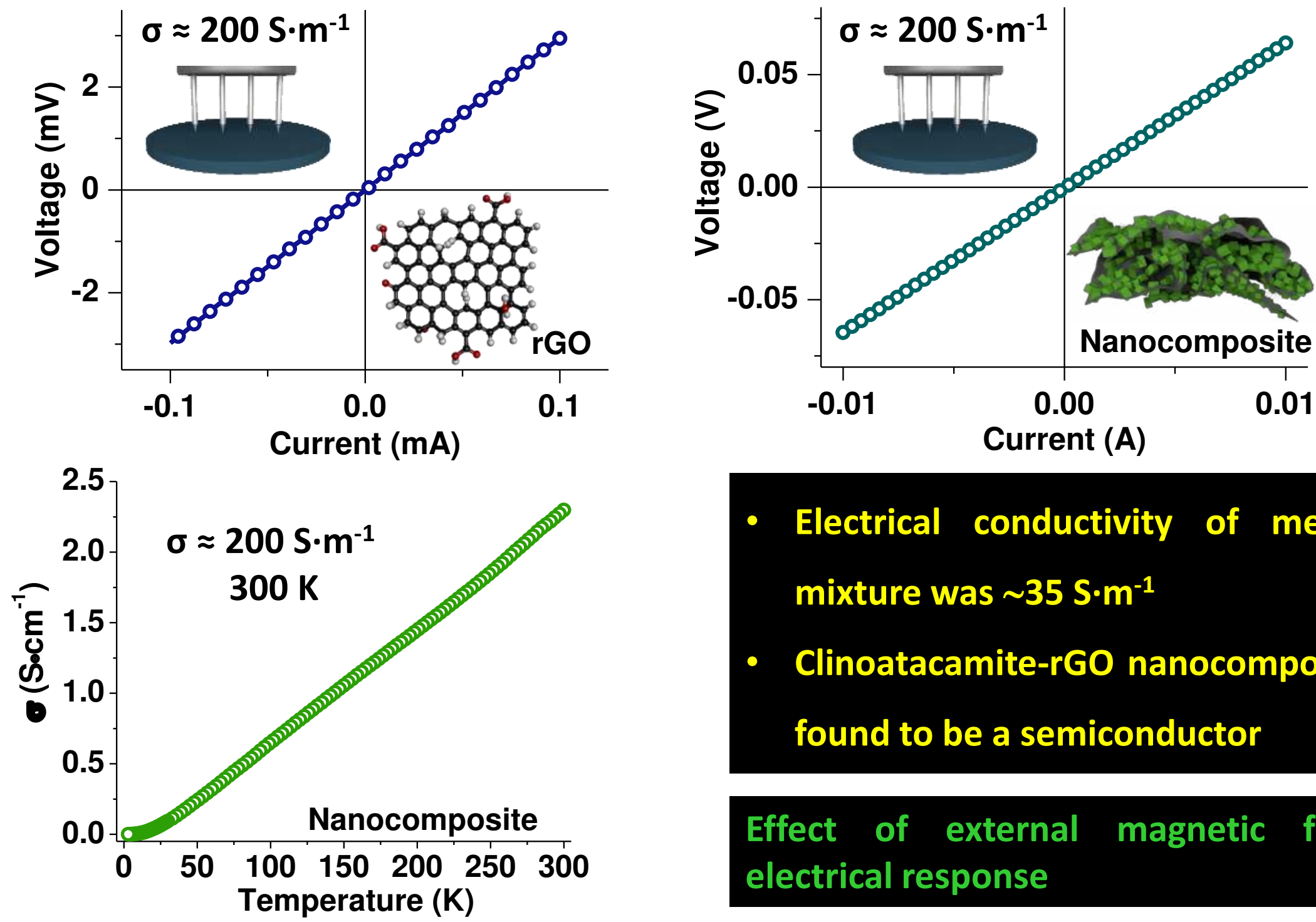

- Electrical conductivity of mechanical mixture was $\sim 35 \mathrm{~S} \cdot \mathrm{m}^{-1}$

- Clinoatacamite-rGO nanocomposite was found to be a semiconductor

Effect of external magnetic field on electrical response 


\section{Summary}

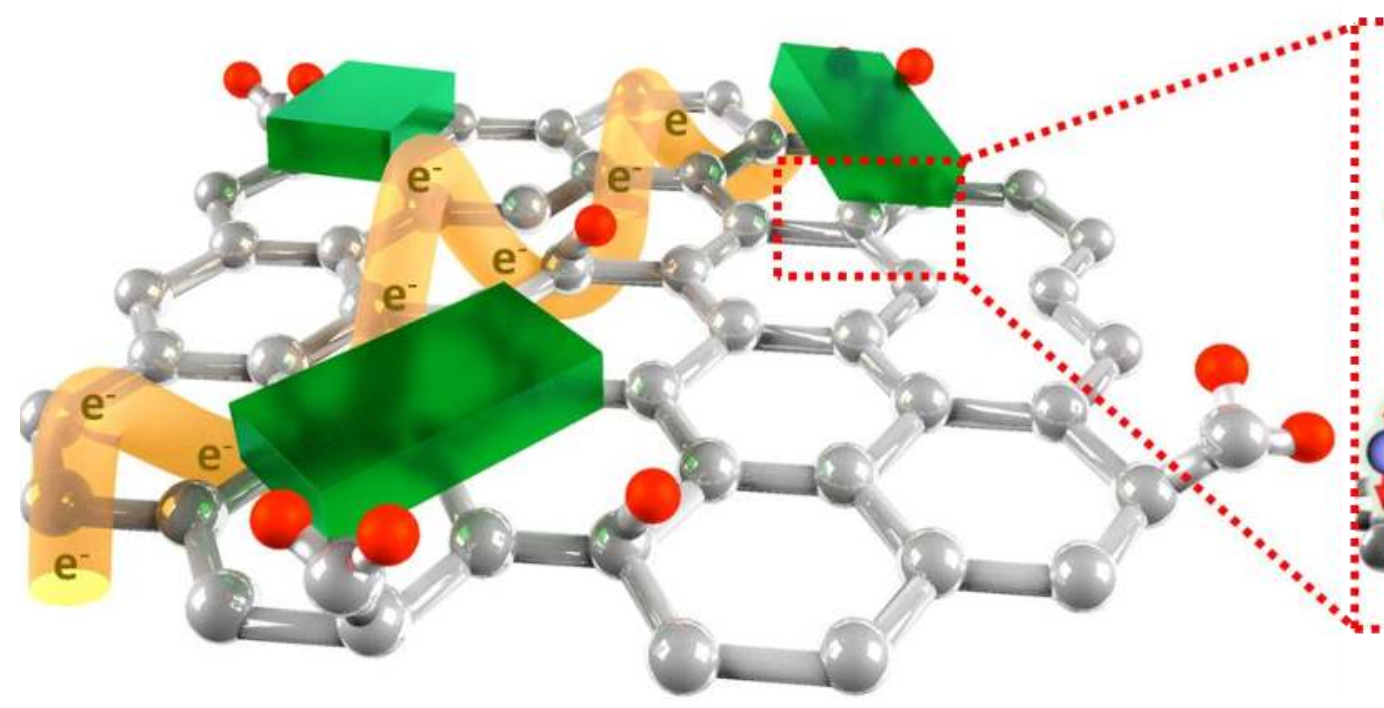

- In situ formation of clinoatacamite onto rGO sheets from using simple oxidation-reduction reaction

$$
\mathrm{Cu}(\mathrm{I})+\mathrm{GO} \longrightarrow \text { Clinoatacamite(Cu(II))-rGO }
$$

- $S=1 / 2$ Kagome-like lattice onto rGO sheets - a new type of magnetic semiconductor

- Scope to extend this approach for other magnetic insulators to synthesize a fundamentally interesting new class of magnetic semiconductors

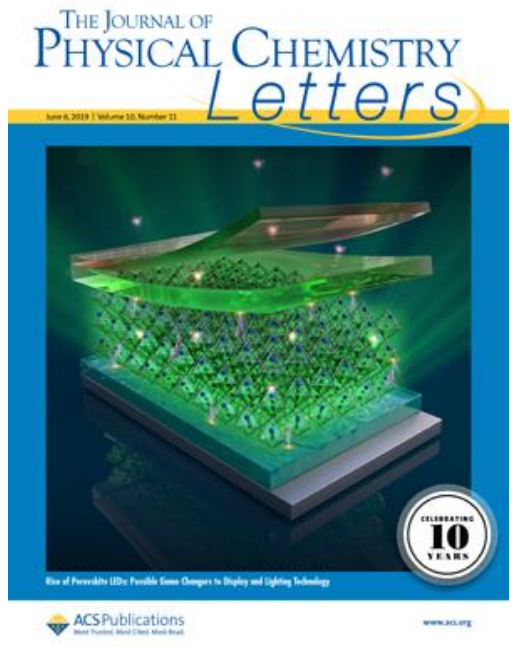

K. Gupta et al.

J. Phys. Chem. Lett. 2019, 10, 2663-2668 

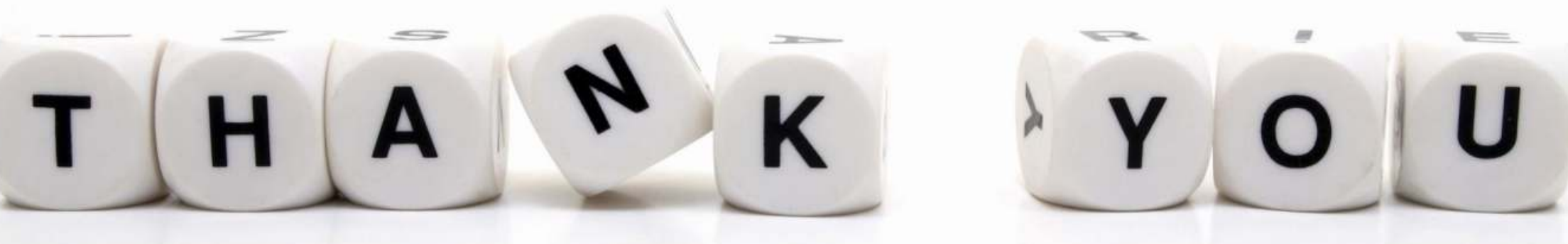